if

TRANS · núm. I9.I · 2015

MONOGRÁFICO · 33-41
Esta contribución pretende dar cuenta de las coordenadas en las que se sitúa el trabajo del intérprete de conferencia en un entorno judicial multilingüe por excelencia, el Tribunal de Justicia de la Unión Europea. Para ello, se parte del marco normativo en el que se inscribe la institución y de aquellas disposiciones que establecen el régimen lingüistico que le es aplicable (desde el Reglamento número 1 del Consejo de 15 de abril de 1958 hasta los distintos Reglamentos de Procedimiento) y que consagran la situación de multilingüismo que caracteriza el trabajo de las tres jurisdicciones que componen la institución Tribunal de Justicia de la Unión Europea: el Tribunal de Justicia, el Tribunal General y el Tribunal de la Función Pública. A continuación, se pasa revista a las características propias de la labor del intérprete en el contexto particular previamente acotado, prestando especial atención a las consideraciones deontológicas, al tipo de usuarios específico, a la función de mediación lingüística en un entorno de "alto contexto compartido" y a la fase de preparación de los asuntos, como etapa clave y diferenciadora en la interpretación que se practica en el Tribunal de Justicia.

palABRAS Clave Tribunal de Justicia de la Unión Europea, Multilingüsimo, Interpretación de conferencias, Preparación de los asuntos

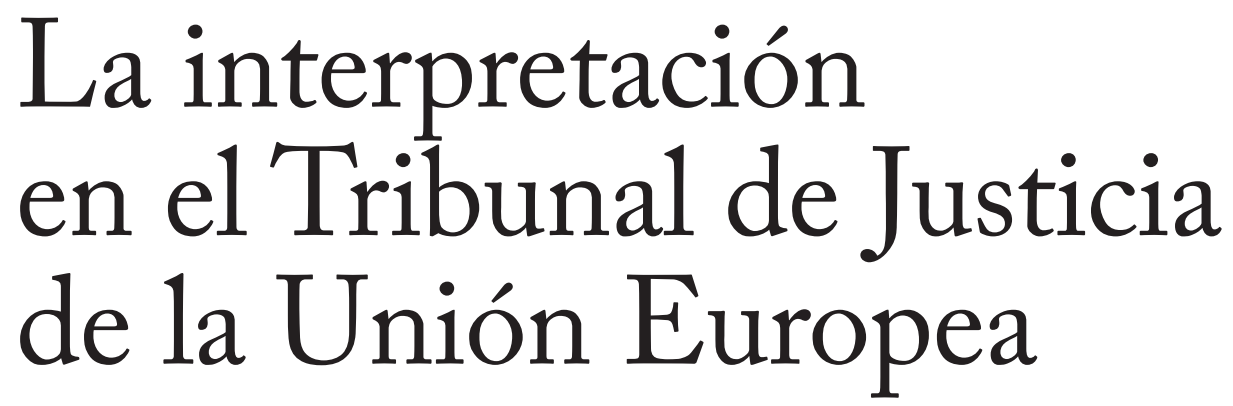

Marina Pascual Olaguíbel ${ }^{*}$

Tribunal de Justicia de la Unión Europea

* Las opiniones expresadas en esta contribución solo comprometen a su autora y no a la institución.

\section{Interpreting at the Court of Justice of the European Union}

The goal of this article is to give an account of the characteristic features of the work of a conference interpreter at the Court of Justice of the European Union: a multilingual legal setting par excellence. The starting point is the institution's regulatory framework, especially the provisions laying down language arrangements (from Regulation No 1 of 15 April 1958 to the various Rules of Procedure). The principle of multilingualism is enshrined therein and underpins the work of the three jurisdictions which make up the Court of Justice of the European Union: the Court of Justice, the General Court and the Civil Service Tribunal.

The main features of the work of the interpreter in this specific context are then reviewed, with particular reference to ethical considerations, types of user, the role of mediation in an environment of "highly-shared context" and the case preparation phase, which is a key and distinguishing feature of interpreting at the Court of Justice.

KEY WORDS Court of Justice of the European Union, Multilingualism, Conference Interpreting, Case preparation 
I. INTRODUCCIÓN: EL TRIBUNAL DE JUSTICIA, UNA INSTITUCIÓN QUE CONFORMA NUESTRA REALIDAD

Cualquier ciudadano español que en el año 20I4 haya tenido un cierto interés por la realidad que le rodea, así como el tiempo y el ánimo de estar informado sobre ella, se habrá detenido en algún momento para analizar hechos como el llamado "céntimo sanitario» ${ }^{\mathrm{I}} \mathrm{y}$ su conformidad con el Derecho de la Unión); se habrá topado, quizá en sus desplazamientos, con que las compañías aéreas gozan de libertad en la fijación de precios, incluidos servicios tales como la facturación de maletas ${ }^{2} \mathrm{y}$, con absoluta seguridad, habrá participado en numerosas conversaciones sobre los desahucios hipotecarios, en las que es harto probable que alguien haya esgrimido esa posibilidad del juez español de declarar, en determinadas circunstancias, abusiva una cláusula de un contrato de préstamo hipotecario. ${ }^{3}$

No he elegido al azar estos asuntos, sino pensando en su calado en términos económicos, o en la manera como conforman nuestra realidad cuando, por ejemplo, hemos de subir a un avión, y, desde luego, en la grandísima importancia que tienen en la vida social, que los hace aún más llamativos.

No son, sin embargo, los únicos asuntos sobre los que se ha pronunciado el tribunal. El número de ellos a los que se ha atendido en las tres jurisdicciones en el año $2013,1587,4$ constituye

\footnotetext{
I Asunto C-82/12, Transportes Jordi Besora, comunicado de prensa

2 Asunto C-487/r2, Vueling Airlines, comunicado de prensa

3 Asunto C-415/II, Mohamed Aziz, comunicado de prensa

4 Informe anual 20I3. Tribunal de Justicia de la Unión Europea. Dato calculado a partir de los $70 \mathrm{or}$ asuntos terminados (2013) del Tribunal de Justicia, 702 asuntos termina-
}

un dato importante para valorar la magnitud del trabajo desempeñado por la institución. Son muchos los que no llegan a las cabeceras de los principales periódicos, aunque resulten esenciales para el funcionamiento de la UE, en ámbitos tan dispares como el espacio de libertad, seguridad y justicia; la protección del medio ambiente y de los consumidores; la libre circulación de personas, de mercancías y capitales; el derecho institucional; las ayudas de Estado; el derecho de competencia; la fiscalidad; la discriminación indirecta por razón de sexo o de edad; o la aproximación de las legislaciones de los Estados miembros en distintas materias como medio de la UE para la consecución de los objetivos de los Tratados de la Unión Europea. Tampoco podemos dejar de mencionar el importante contencioso sobre la marca comunitaria y todo lo que tiene que ver con el Estatuto de Funcionario de la Unión Europea.

\section{EL MULTILINGÜISMO, ELEMENTO CARACTERÍSTICO Y DIFERENCIADOR}

Una vez esbozada la complejidad, el interés y el volumen del contencioso, cabe considerar, a diferencia de lo que ocurre en los órganos jurisdiccionales nacionales, el multilingüismo como elemento diferenciador en todas las fases de los procedimientos ante el Tribunal de Justicia. ${ }^{5}$

Antes de analizar dicha situación, convendría delimitar cuál es la posición del francés en el Tribunal de Justicia, pues se trata de la lengua que sirve tradicionalmente en la institución, de modo oficioso, como lengua vehicular, al tratar-

dos (20I3) del Tribunal General y i84 asuntos terminados (2013) del Tribunal de la Función Pública

5 En el sentido del artículo I9.I del Tratado de la Unión Europea ( El Tribunal de Justicia de la Unión Europea comprenderá el Tribunal de Justicia, el Tribunal General y los tribunales especializados), entendemos que la expresión Tribunal de Justicia engloba las tres jurisdicciones. 
se del «idioma de deliberación»; lo cual explica y justifica que todos los documentos de los expedientes se traduzcan a ese idioma. Dicho lo cual, abandonado el ámbito de la deliberación interna, en el desarrollo de los procedimientos, la institución trabaja en un régimen particularmente respetuoso con el multilingüismo, especialmente vinculado a los derechos de defensa y a un juicio equitativo.

\section{I. La cuestión prejudicial como ejemplo de procedimiento multilingüe}

Quizá donde se haga más patente este rasgo sea en las cuestiones prejudiciales. ${ }^{6}$ Se trata de un tipo de procedimiento que se ha dado en llamar «diálogo entre jueces», en el que los órganos jurisdiccionales de los Estados miembros se dirigen al Tribunal de Justicia para comprobar la conformidad de una normativa nacional con el Derecho de la Unión, cuya aplicación resulta necesaria para resolver un determinado asunto. La respuesta que da el Tribunal de Justicia adopta la forma de sentencia o de auto motivado y vincula en la resolución del litigio nacional al órgano jurisdiccional que ha planteado la cuestión prejudicial. Vincula también a todos los órganos jurisdiccionales, huelga decirlo, de todos los Estados Miembros de la Unión que conozcan de un litigio similar. En el procedimiento iniciado ante el Tribunal de Justicia pueden participar las partes del litigio principal, además de los Estados Miembros y de la Comisión Europea en su calidad de amicus curiae. Una vez concluida la fase escrita, en la celebración de la vista el multilingüismo es la herramienta imprescindible para facilitar ese

6 La publicación divulgativa «E1 Tribunal de Justicia: composición, competencias y procedimientos» ofrece una panorámica general de cuáles son los tipos de procedimiento ante el Tribunal de Justicia : <http://curia.europa. eu/jcms/upload/docs/application/pdf/20I2-05/cjue_es.pdf> diálogo. La lengua de procedimiento será la de la jurisdicción nacional que plantea la cuestión prejudicial, lo que hace que, en el plano de lo concreto, las partes del procedimiento nacional que ha dado origen al asunto ante el Tribunal de Justicia intervengan en su propio idioma. Los Estados Miembros que decidan intervenir están autorizados a hacerlo en el suyo para entenderse con los miembros de la sala. Y estos los escucharán gracias a la interpretación simultánea que se convierte en el revés de la moneda del multilingüismo: sin la interpretación no puede hacerse efectivo el multilingüismo en la fase oral. Precisamente para servir a ese multilingüismo se ha desarrollado un sistema de interpretación tan cuidadoso.

\subsection{Desarrollo normativo del multilingüismo}

Es, pues, en esta condición peculiar del multilingüismo donde encontramos la razón de ser del trabajo de los intérpretes en el Tribunal de Justicia, situación reconocida en el artículo 342 del Tratado de Funcionamiento de la Unión Europea, ${ }^{7}$ fijada inicialmente en el Reglamento $\mathrm{I} / 58^{8}$ y regulada detalladamente en el Reglamento de Procedimiento de la institución, en su capítulo octavo, Del régimen lingüistico. ${ }^{9}$

7 «El régimen lingüístico de las instituciones de la Unión será fijado por el Consejo mediante reglamentos, por unanimidad, sin perjuicio de las disposiciones previstas en el Estatuto del Tribunal de Justicia de la Unión Europea».

8 En realidad son dos los Reglamentos I/58: uno, válido para la CEE y el otro para la Comunidad Europea de la Energía Atómica CEEA, cada uno de los cuales fijaba un régimen lingüístico idéntico para las respectivas comunidades.

9 Esta disposición encuentra su parangón en el artículo 35 del Reglamento de Procedimiento del Tribunal General y que está siendo objeto de revisión en el otoño de 20I4. En el caso del Reglamento de Procedimiento del Tribunal de la Función Pública no encontramos una regulación equiparable acerca del régimen lingüístico. Esta aparente laguna no lo es tal, ya que viene suplida en el artí- 
Viene al caso traer a colación su artículo 36 que, a pesar de ser una mera enumeración, es donde encontramos el origen del multilingüis- mo tal y como lo entiende y aplica la institución.

Artículo 36 Lenguas de procedimiento

Las lenguas de procedimiento serán el alemán, el búlgaro, el checo, el croata, el danés, el eslovaco, el esloveno, el español, el estonio, el finés, el francés, el griego, el húngaro, el inglés, el irlandés, el italiano, el letón, el lituano, el maltés, el neerlandés, el polaco, el portugués, el rumano y el sueco.

Es la lengua de procedimiento —una de las 24 enumeradas - el primer dato que hemos de tener en cuenta para entender cómo va a determinarse en la práctica, caso por caso, la conformación del equipo de interpretación. El segundo será la lengua de los Estados Miembros que intervengan. La composición de la sala (Jueces y Abogados Generales) y su «perfil lingüístico» será el tercer elemento crucial para confeccionar el equipo. El último factor viene dado por el idioma de los grupos de visitantes que, previamente acreditados, asistan a las vistas.

\section{LA INTERPRETACIÓN, CONDICIÓN IMPRESCINDIBLE PARA GARANTIZAR EL MULTILINGÜISMO}

\section{I. La preparación del asunto, clave del éxito}

En este contexto se inserta la labor de los intérpretes del Tribunal de Justicia, que no es otra que facilitar la comunicación en las vistas que se celebran en las tres jurisdicciones de la institución, ${ }^{\text {IO }}$ para lograr que los participantes

culo 7.2 del Estatuto del Tribunal de Justicia que establece que «las disposiciones relativas al régimen lingüístico del Tribunal General se aplicarán al Tribunal de la Función Pública».

ro En el año 2013 se celebraron 629 vistas y otras reuniones con interpretación. (agentes de los Estados Miembros, representantes de las partes, agentes de las instituciones, miembros de la sala) puedan comunicarse en pie de igualdad en un entorno multilingüe.

Pero, ¿a quiénes nos referimos cuando hablamos de «los intérpretes» del Tribunal de Justicia ${ }^{\text {Ir? }}$ Estamos hablando de una realidad muy concreta, ya que la Dirección de Interpretación, garante del multilingüismo en la fase oral, cuenta con tan solo unos 75 intérpretes en plantilla para 22 cabinas. $^{\mathrm{I2}}$ Se trata de un servicio muy especializado, pero de dimensiones reducidas, lo que hace que asiduamente se recurra a la lista común de intérpretes freelance acreditados por la Unión Europea que asumen una gran parte de la carga de trabajo.

\subsection{Intérpretes de conferencia en un contexto jurisdiccional}

Tanto funcionarios como freelance son intérpretes de conferencia «facultados» (en el caso de unos, por la realización de un concurso oposición, ${ }^{13}$ en el caso de otros por la superación de las pruebas interinstitucionales de acreditación ${ }^{\mathrm{I} 4}$ ) para trabajar en un entorno judicial altamente especializado. Esto quiere decir que

II A diferencia de lo que ocurre con los juristas lingüistas, a los intérpretes no se les exige una formación universitaria jurídica pero sí una formación universitaria específica en interpretación de conferencias.

I2 En la actualidad no hay intérpretes permanentes en el caso del maltés o del irlandés lo cual no significa que la Dirección no esté capacitada para ofrecer interpretación en esos idiomas, si se diese la necesidad.

r3 En la actualidad, todos los procesos de selección de personal, incluidos los concursos-oposición los realiza, de manera centralizada, la Oficina Europea de Selección de Personal (EPSO), de los cuales se informa en la página <http://europa.eu/epso/apply/jobs/perm/20I3/interpreters/ index_en.htm>

${ }_{14}$ En la siguiente dirección se encuentra la información acerca de cómo acreditarse como intérprete freelance o autónomo ante las instituciones de la UE: <http://europa. eu/interpretation/accreditation_en.htm> 
dominan la técnica de la interpretación simultánea, utilizada en todas las vistas celebradas en Luxemburgo, que conocen a fondo sus idiomas de trabajo, que están familiarizados con la manera que tiene el derecho de decir las $\operatorname{cosas}^{15} \mathrm{y}$ de hacerlas, es decir que son capaces de expresarse en cabina con el mismo registro y la misma precisión con que lo hacen los juristas en la sala y que conocen el, llamémoslo, «ritual» que se sigue en el desarrollo de una vista, con datos tan aparentemente banales, pero tan interesantes para el trabajo del intérprete, como dónde se sienta cada uno de los miembros de la sala, qué orden de intervenciones se va a producir y, conociendo ese orden, saber de qué tipo de intervenciones se trata en cada momento (informes orales, turno de preguntas, réplicas de las partes).

Una vez señalada la vista, se comunica la información al Servicio de Interpretación que, sabiendo cuál es la lengua de procedimiento, quiénes son los miembros de la sala, los Estados Miembros que intervienen y, en su caso, el idioma de los visitantes va a confeccionar un equipo ad hoc para la vista. Para ello, se determina en primera instancia qué idiomas se necesitan (desde el punto de vista de cuáles son aquellos en que se va a poder intervenir y hacia cuáles se va a interpretar). A partir de esta información, el servicio de planning «rellenará» cada cabina con los intérpretes que mejor encajen, por su combinación lingüística, con el -llamémoslo así- arreglo lingüístico de la vista. Conviene no olvidar, como ya hemos señalado en otra ocasión (Pascual Olaguíbel 2oro: 8), que lo que hemos

I5 «Un jurista ha de conocer bien la propia lengua y las peculiaridades del lenguaje jurídico porque de su correcta expresión depende, en muchos casos, la justa solución de los problemas. De algunos de ellos ha partido la iniciativa de emplear con propiedad, claridad y sencillez la lengua.» (Jiménez Yáñez 20I3: 44). dado en llamar «arreglo lingüístico» (por evitar confusiones con el término «régimen lingüístico») varía enormemente, según la vista de que se trate, mostrando una cierta asimetría, si lo comparamos con otras instituciones internacionales en las que los idiomas objeto de interpretación son el resultado de una decisión política previa.

\section{4. Consideraciones prácticas. Programa, hoja de equipo, durée des plaidoiries}

Pero, ¿qué hace el intérprete cuando ve que se le ha señalado determinada vista en su programa? Cabe indicar, para empezar, que el intérprete puede consultar el programa desde su despacho unas ocho semanas antes, por más que esté supeditado a cambios y que en la práctica se cierre y considere definitivo tan solo al mediodía del viernes anterior a la semana pertinente.

$\mathrm{La}$ información que recibe el intérprete incluye la referencia del asunto, con una signatura distinta según la jurisdicción de que se trate, que incluye en un formato comprimido datos acerca de ella, del número del asunto y del año en el que fue introducido el recurso. Así, el asunto C-531/r3. También se le informa acerca de dónde se celebrará la vista, dato esencial, ya que es variable y resulta importante que el intérprete llegue con antelación a la vista y pueda encontrar con facilidad la sala: en este caso se celebró el 4 de septiembre de $20{ }_{4}$ a las I4 horas en la Sala de vistas III de la sexta planta. Otro elemento pertinente que obtendrá solo con una ojeada es la lengua de procedimiento, en este caso alemán, y la "hoja de equipo», es decir qué intérpretes más han sido asignados a esa reunión. Si trabaja con un colega freelance acreditado, se tendrá que ocupar de proporcionarle el expediente, mientras prepara su propia copia, imprimiéndoselo y dejando en secretaría un juego de los escritos procesales, del informe para la vista (documento interno que resume el 
marco normativo y fáctico del asunto) y de la legislación pertinente. Para hacerlo, el intérprete tiene acceso a la base de datos interna del Tribunal de Justicia donde se almacenan los escritos procesales de cada uno de los asuntos. En la actualidad es muy frecuente recurrir a tabletas y demás dispositivos como apoyo para el trabajo de los intérpretes en cabina. Para un intérprete al que se le asignan, como promedio, tres vistas semanales, el formato digital ofrece ventajas inigualables: facilidad de acceso, comodidad para trasladar expedientes a la sala de vistas sin tener que acarrear volúmenes ingentes de papel y un nada desdeñable ahorro institucional, así como un importante beneficio ecológico.

Para entender la importancia que tiene la preparación de los asuntos en el Tribunal de Justicia debemos decir que es la única institución de la UE que contrata al freelance un día más para que dedique esa jornada de trabajo adicional previa al día de la vista a estudiar in situ el expediente, al que tendrá acceso en un despacho similar al que utilizan los intérpretes permanentes en el edificio del Tribunal.

Usando una imagen anglosajona que tiene una cierta belleza, podríamos decir que para el intérprete la vista no es más que la punta de un iceberg; la culminación de un proceso largo de preparación que habrá iniciado con antelación, el expediente, decidiendo qué documentos son pertinentes y, en su caso, de los textos legislativos en las diferentes versiones lingüísticas. En nuestro ejemplo anterior ( $\left.\mathrm{C}-53^{\mathrm{I}} / \mathrm{s} 3\right)$, el intérprete se sitúa ante una cuestión prejudicial planteada por el Verwaltungsgerichtshof austríaco. Las cuestiones prejudiciales que el tribunal nacional plantea al Tribunal de Justicia se publicarán en el Diario Oficial, ${ }^{16}$ para que todos los Estados

\footnotetext{
I6 http://eur-lex.europa.eu/legal-content/ES/TXT/ $\mathrm{PDF} /$ ?uri=CELEX:620I3 $\mathrm{CN}$ 053 1 \&qid $=1409510810743 \&$ fro $\mathrm{m}=\mathrm{IT}$
}

Miembros tengan acceso a su contenido y puedan decidir si participan o no en el asunto.

Llegados a este punto, un posible esquema de preparación puede ser el siguiente. Tras una primera lectura del auto de remisión en español, el intérprete identifica el contenido de la cuestión prejudicial y el área temática en la que esta se sitúa: en nuestro caso en el campo del medio ambiente, más concretamente en el terreno de las evaluaciones de impacto ambiental, tras la realización de pruebas de extracción de gas natural en un municipio austríaco. En cuanto a los referentes legislativos, se percata de que son dos los referentes legislativos pertinentes: la normativa nacional, en este caso la ley austríaca en la materia (Umweltverträglichkeitsprüfungsgesetz) y la normativa comunitaria (Directiva 97/ri/CE del Consejo de 3 de marzo de 1997 por la que se modifica la Directiva 85/337/CEE relativa a la evaluación de las repercusiones de determinados proyectos públicos y privados sobre el medio ambiente).

A medida que avanza en la lectura de los escritos de las partes y en paralelo a la comprensión conceptual, va realizando un vaciado terminológico de los textos, cuyo resultado se plasmará, por ejemplo, en un glosario en el que se recojan equivalentes funcionales tales como los tres que figuran a continuación:

Förderung $\Rightarrow$ Extracción

Wirtschaftlichkeit $\Rightarrow$ Viabilidad económica

Umweltverträglichkeitsprüfung $\Rightarrow$ Evaluación de impacto ambiental

Es aquí donde comienza, si no lo ha hecho antes, el trabajo en equipo, tanto para determinar una línea terminológica homogénea como para, a partir del documento Durée des plaidoiries, hacer un reparto del trabajo, teniendo fundamentalmente en cuenta la combinación lingüística de cada intérprete. En el caso que nos ocupa fueron 
seis las partes que intervinieron en la vista. Ese documento establece el orden de intervención de las partes, el nombre de sus representantes, es decir quiénes va a intervenir oralmente, y la duración del tiempo atribuido a cada uno de los oradores. Los intérpretes deciden quién interpreta a quién, por lo general siguiendo un orden alternativo, lo cual les permite centrar su preparación en los textos que les correspondan. Así pueden dedicarles más tiempo y preparar de forma más concienzuda las alegaciones de las partes que les toque interpretar.

Llegado el día de la vista, y como hemos señalado en otra ocasión, «el intérprete antes de entrar en cabina conoce el «hilo argumental» del asunto, sabe cuál es la posición (con todos sus matices) que defiende cada una de las partes y domina los términos jurídicos y los específicos del tema» (Pascual Olaguíbel 20ro: 29). Añadiríamos que se ha familiarizado también con la jurisprudencia alegada por las partes, $y$ que conoce los términos de los apartados que se citan en la versión española. En nuestro ejemplo, se citaron varios asuntos, curiosamente uno de ellos surgido con una cuestión prejudicial española (C-I42/o7 Ecologistas en acción/ CODA). Lo importante entonces es estar en cabina, como mínimo, media hora antes del inicio. Se trata de un tiempo esencial para poder instalarse, comprobar el sonido, preparar los dispositivos electrónicos, accediendo a la red wi-fi $\mathrm{y}$ al expediente, $\mathrm{y}$ sobre todo para no perder la posibilidad de leer los textos de los informes orales que lleguen en el último momento. Con relación a los informes orales, son tres las posibilidades: que se hayan enviado con antelación al Servicio de Interpretación y que se hayan distribuido entre el equipo la tarde anterior, ${ }^{17}$ que

${ }^{17}$ Huelga decir que los intérpretes están obligados por el más estricto secreto profesional y el mayor respeto de la confidencialidad. Así, en las «Instrucciones prácticas» del el jefe del equipo los obtenga antes de la vista y haga copias para los colegas, o que no haya texto y hablen libremente. ${ }^{18}$

Este tiempo previo será, por lo tanto, de un 39 grandísimo provecho para una lectura rápida de los informes orales que hayan llegado, que sobre la base de la preparación anterior permitirán la identificación de los escollos, hacer anotaciones, buscar referencias en los textos legislativos y en la jurisprudencia e incluso, como podría hacer un deportista antes de una competición, concentrarse y relajarse antes de que comience la interpretación propiamente dicha.

Una vez que empieza la vista, se seguirá el reparto previamente realizado entre los intérpretes. Tras concluir los informes orales, basados por lo general en textos escritos, muy densos, a menudo leídos a una gran velocidad, se inicia el turno de preguntas, donde el intérprete trabajará con material puramente oral, sin apoyatura escrita. Tras el turno de preguntas, la norma es conceder a las partes, como broche final de la vista, otro turno de réplica, que carecerá también, obviamente, de soporte escrito.

\section{A MODO DE CONCLUSIÓN}

Con este artículo hemos pretendido dar cuenta de la manera en que se desarrolla el trabajo de

Tribunal general se recuerda expresamente a las partes que «[S]e garantiza la confidencialidad de las notas sobre los informes orales que se hayan remitido».

I8 A pesar de ser la opción menos practicada, es la preferida de los intérpretes y de la institución. Así se recoge en las «Instrucciones prácticas» de las distintas jurisdicciones. Así, el Tribunal de Justicia observa que «[E]n la vista propiamente dicha, se desaconseja, no obstante, leer un escrito. El Tribunal de la Función Pública, por su parte, aborda las particularidades de la interpretación simultánea, señalando que, «en los asuntos que requieren interpretación simultánea, generalmente es preferible hablar libremente utilizando notas que leer un escrito». 
los intérpretes de conferencia en el Tribunal de Justicia. Llegados a este punto, nos atrevemos a hablar de una manera de hacer «artesanal» que, sin desdeñar el recurso a las más avanzadas tecnologías, se plantea como una labor meticulosa, delicada y concienzuda, en la que el tiempo que transcurre entre la apertura y el cierre del micrófono en una vista determinada es, como ocurre con los artesanos, solo una fracción de todas las tareas preparatorias que se han llevado a cabo previamente y que son la clave del éxito, es decir de la posibilidad de lograr cumplir con el cometido de que la comunicación sea fluida en un entorno multilingüe y altamente especializado.

\section{REFERENCIAS}

Comunicado de prensa «Sentencia en el asunto C-487/ı2. Vueling Airlines S.A./Instituto Galego de Consumo de la Xunta de Galicia. Al obligar a las compañías aéreas a transportar el equipaje facturado del pasajero sin suplemento de precio, la normativa española es contraria al Derecho de la Unión», Servicio de Prensa e Información, Tribunal de Justicia <http://curia.europa.eu/jcms/ upload/docs/application/pdf/20I4-o9/cpi4OI27es. pdf $>$ [Consulta: 29 septiembre 20I4]

Comunicado de prensa «Sentencia en el asunto C-82/12. Transportes Jordi Besora S. L./ Generalitat de Catalunya. El impuesto sobre ventas minoristas de determinados hidrocarburos es contrario al Derecho de la Unión», Servicio de Prensa e Información, Tribunal de Justicia $<\mathrm{http} / /$ curia.europa.eu/jcms/upload/docs/application/pdf/2OI4-02/cpr40022es.pdf> [Consulta: 29 septiembre 20I4]

Comunicado de prensa «Sentencia en el asunto C-4I5/II, Mohamed Aziz/Catalunyacaixa. La normativa española, que impide al juez que es competente para declarar abusiva una cláusula de un contrato de préstamo hipotecario suspender el procedimiento de ejecución hipotecaria iniciado por otra vía, es contraria al Derecho de la Unión», Servicio de Prensa e Información, Tribunal de Justicia, <http://curia.europa.eu/jcms/upload/ docs/application/pdf/2013-03/cpr3003oes.pdf> [Consulta: 29 septiembre 20I4]

Instrucciones Prácticas a las Partes en los Procedimientos ante el Tribunal General de 24 de enero de 2012 (DO L 68/40, de 7.3.2.oI2) <http://eur-lex. europa.eu/LexUriServ/LexUriServ.do?uri=OJ:L :2012:068:0023:0041:ES:PDF> [Consulta: 29 septiembre 20I4]

Instrucciones Prácticas a las Partes sobre los Asuntos sometidos al Tribunal de Justicia de 25 de noviembre de 20I3 (DO L 3I/I, de 3I.I.2OI4) $<$ http://eur-lex.europa.eu/legal-content/Es/TXT/ PDF/?uri=OJ:L:20I4:O31:FULL\&from=ES> [Consulta: 29 septiembre 20I4]

Instrucciones Prácticas a las Partes sobre el Procedimiento ante el Tribunal de la Función Pública de la Unión Europea de 2I de mayo de 20I4 (DO L 206, de I4.07.20I4) <http://eur-lex.europa.eu/ legal-content/ES/TXT/PDF/?uri=OJ:L:20I4:206:F ULL\&from=ES> [Consulta: 29 septiembre 20I4]

Jiménez Yáñez, R. M. (2013) «ंSe puede enseñar a persuadir a los alumnos de Derecho con el metadiscurso? Una propuesta docente». Revista de Llengua i Dret, n. 59. <http://revistes.eapc. gencat.cat/index.php/rld/article/view/ro.243620.8030.02.3/244> [Consulta: 29 septiembre 20I4]

Nota «Languages and interpreting at the Court of Justice of the European Union in Luxembourg» rev I/20Io-EN <http://eulita.eu/sites/default/ files/Interpreting\%2oat\%2othe\%2oCourt\%20 of\%2oJustice\%2oof\%2othe\%2oEU.pdf> [Consulta: 29 septiembre 20I4]

Pascual Olaguíbel, M. (2010): «La interpretación al español en el Tribunal de Justicia de la Unión Europea (TJ): algunos apuntes», Puntoycoma, n II7, marzo/abril/mayo 20Io. <http://ec.europa.eu/ translation/bulletins/puntoycoma/II $/$ pyciI7II_ es.htm> [Consulta: 29 septiembre 20I4]

Reglamento I/58 de 15 de abril de 1958 , por el que se fija el régimen lingüístico de la Comunidad Económica Europea y el Reglamento no I, de I5 de abril de 1958, por el que se fija el régimen lingüístico de la Comunidad Europea de la Energía Atómica, Diario Oficial I7 de 6.Io.1958, p. $385 / 58$.

Reglamento de Procedimiento del Tribunal de Justicia, de 25 de septiembre de 2012 (DO L 265, de 29.9.20I2), en su versión modificada el I8 de junio de 2013 (DO L I73, de 26.6.20I3, p. 65), <http:// curia.europa.eu/jcms/upload/docs/application/ 
pdf/2or2-Io/rp_es.pdf>[Consulta: 29 septiembre 2OI4]

Reglamento de Procedimiento del Tribunal de Justicia, de 25 de septiembre de 2012 (DO L 265, de 29.9.2012), en su versión modificada el I8 de junio de 2013 (DO L I73, de 26.6.20I3, p. 65), <http:// curia.europa.eu/jcms/upload/docs/application/ pdf/20I2-Io/rp_es.pdf> [Consulta: 29 septiembre 2OI4]

Reglamento de Procedimiento del Tribunal de la Función Pública, de 2I de mayo de 20I4 (DO L 206, de 14.07.2014) <http://eur-lex.europa.eu/ legal-content/ES/TXT/PDF/?uri=OJ:L:2OI4:206:F ULL\&from=ES > [Consulta: 29 septiembre 20I4]

Reglamento de Procedimiento del Tribunal General, de 2 de mayo de i99i (Do L I36, de 30.5.I991, p. I, con corrección de errores en DO L 3I7, de I9.II.I99I, p. 34), <http://curia.europa.eu/jcms/ upload/docs/application/pdf/2008-o9/txt7_2008- 09-25_10-00-8_829.pdf> [Consulta: 29 septiembre 20I4]

Tratado de Funcionamiento de la Unión Europea, versión consolidada, en <http://eur-lex.europa.eu/ legal-content/ES/TXT/PDF/?uri=CELEX:I2OI2E/ TXT\&from=EN> [Consulta: 29 septiembre 20I4] Tribunal de Justicia de la Unión Europea (2010) El Tribunal de Justicia. Composición, competencias y procedimientos, Luxemburgo: Oficina de Publicaciones de la Unión Europea, 2010, <http:// curia.europa.eu/jcms/upload/docs/application/ pdf/20r2-05/cjue_es.pdf> [Consulta: 29 septiembre 20I4]

Tribunal de Justicia de la Unión Europea (2014) Informe Anual 2013, Luxemburgo: Oficina de Publicaciones de la Unión Europea, 20I4, <http:// curia.europa.eu/jcms/upload/docs/application/ pdf/20I4-06/qdagi40oresc.pdf> [Consulta: 29 septiembre 20I4] 